\title{
Aprendizaje de las competencias comunicativas atenuantes en el aula de E/LE
}

DANIEL SECCHI

Universitat de València / Università degli studi di Cagliari

secchi@unica.it

Resumen: El objetivo de este trabajo es presentar una actividad didáctica que ha sido llevada a cabo en el aula de E/LE con estudiantes franceses de nivel B1/B2. El primer objetivo de la actividad es hacer reflexionar a los estudiantes sobre sus conocimientos pragmáticos previos. En segundo lugar, a través de la explotación de un fragmento de corpus discursivo oral se pretende analizar y averiguar las capacidades atenuantes de los discentes con el fin de mejorar sus destrezas comunicativas atenuantes. El fin último de la actividad es que los estudiantes aprendan a reconocer y usar la atenuación como un recurso estratégico al que se puede recurrir en diferentes contextos y en función de los fines comunicativos que se pretenden lograr.

Palabras clave: atenuación, corpus discursivo oral, E/LE, destrezas comunicativas atenuantes

\section{Learning communication and mitigation skills in S/SL class}

Abstract: In this work we want to share a didactic activity that has been carried out in a class of Spanish as a Second Language (S/SL) with French students of level B1/B2. The first objective of the activity is to make the students reflect on their previous pragmatic knowledge. Secondly, through the use of a fragment extracted from an oral discursive corpus, we aim to analyze and find out the mitigating abilities of the students in order to improve their skills while communicating and attenuating. The ultimate goal of the activity is for students to learn to recognize and use attenuation as a strategy to be used in different contexts and according to the purpose they intend to achieve in each situation.

Key words: mitigations, corpus of oral conversations, Spanish as a Second Language (S/SL), communication and mitigation skills

\section{Introducción}

En las últimas décadas han sido muchos los estudios enfocados a la enseñanza de $\mathrm{E} / \mathrm{LE}$, aunque todavía hay algunos aspectos de disciplinas como la pragmática que siguen siendo inexploradas e inexplotadas. Nos referimos concretamente a la enseñanza de la atenuación en el aula de E/LE. A pesar de que se hayan realizados varios trabajos sobre atenuación ${ }^{1}$, tanto de hablantes nativos españoles como estudios contrastivos de hablantes de español y de otras lenguas, sigue habiendo una carencia de actividades didácticas diseñadas para ser llevada al aula con el fin de desarrollar las competencias pragmáticas de uso de la atenuación en estudiantes de E/LE.

\footnotetext{
${ }^{1}$ Se remiten al proyecto Es.Var.Atenuación (Albelda et alii 2014) y a los trabajos de Ballestero Martí
} (2002), Félix-Brasdefer (2008), Conteras Fernández (2012), Briz y Albelda (2013), entre otros. 
Si es cierto que la enseñanza de contenidos pragmáticos significa necesariamente la contextualización de los hechos lingüísticos, también es cierto que hoy en día esta tarea no debería ser un obstáculo, puesto que los corpus discursivos reales constituyen una herramienta muy valiosa que permite su desarrollo. Emplear corpus discursivos reales como herramientas en la didáctica de E/LE, y del español como segunda lengua (E/L2), permite que los estudiantes adquieran y/o mejoren sus habilidades discursivas en español, con el fin de poder desenvolverse en contextos reales de habla al igual que los nativos. Así pues, los corpus orales reales, como el Val.Es.Co. (2002) y C-ORAL-ROM (2005) constituidos por muestras de habla que no suelen estar presentes en los manuales de E/LE, representan una herramienta imprescindible para el desarrollo de las competencias comunicativas de los aprendientes de E/LE, especialmente cuando los estudiantes no se encuentran en un contexto de inmersión lingüística en el país de la lengua meta. De acuerdo con Canale (1983: 32-43), el docente debería actuar como mediador para facilitar un aprendizaje consciente en los aprendientes, en el que se transmitan las competencias y subcompetencias comunicativas necesarias para la autonomía comunicativa de los estudiantes.

Según Briz (2003: 45), el hablante tiene que ser formado en el uso del fenómeno de la atenuación "para poder utilizarlo y entenderlo como herramienta fundamental para el progreso normal de la conversación». En la misma línea de pensamiento, FélixBrasdefer (2004: 8) afirma que los discentes de una lengua extranjera, o de una segunda lengua, no necesitan solamente un aprendizaje gramatical, sino también adquirir los conocimientos pragmalingüísticos y socioculturales de la cultura meta. Si los discentes no aprenden qué estrategias atenuantes podrían emplearse para mitigar una posible amenaza a la imagen propia o de sus interlocutores, no solamente se arriesgan a que se dificulte el acuerdo comunicativo, sino que puede haber un fracaso conversacional. Para ello, la enseñanza-aprendizaje de la atenuación en el aula de E/LE permite a los estudiantes adquirir

una estrategia pragmática (comunicativa) originada por necesidades de imagen y dirigida a mitigar y minimizar la intensidad de lo que se expresa reduciendo la fuerza ilocutiva del acto de habla, y en ocasiones, a través de mecanismos de lenguaje vago por los que se difumina o minimiza el contenido proposicional.

Albelda (2016: 32)

Así pues, de acuerdo con Albelda y Fernández (2006), los corpus orales y sus transcripciones constituyen una buena herramienta para el diseño de materiales de E/LE y E/L2, puesto que a través de estos es posible enseñar tanto la lengua como la cultura.

La actividad didáctica que aquí proponemos ha sido diseñada para un grupo de estudiantes universitarios aprendices de E/LE con un nivel de español entre un nivel B1B2 (MCER 2001). Los estudiantes que componen el grupo son 14, de los cuales 9 son mujeres y 5 son hombres. La duración de la actividad es de dos horas y para su diseño han sido definidos los objetivos generales y específicos que se detallan a continuación.

\section{Punto de partida para la creación de la actividad didáctica}

\subsection{Objetivos generales y específicos para la creación de la actividad didáctica}


Objetivos generales:

Averiguar las competencias lingüísticas atenuantes del grupo de discentes analizado y mejorar sus destrezas comunicativas atenuantes. Para ello ha sido necesario plantearnos algunas preguntas de investigación:

a. ¿Los estudiantes conocen y son capaces de elegir y aplicar las tácticas atenuantes adecuadas al contexto y a los interlocutores con los que interactúan?

b. ¿Los estudiantes saben cómo lograr sus metas conversacionales sin dañar la imagen del otro o su propia imagen (yo del propio hablante)?

c. ¿Los estudiantes son conscientes que el empleo de elementos atenuantes contribuye a agilizar sus destrezas conversacionales con el fin de poder desenvolverse mejor en contextos comunicativos reales al igual que los nativos?

Objetivos específicos:

a. Hacer reflexionar a los estudiantes sobre sus capacidades, carencias o incapacidades atenuantes.

b. Activar un aprendizaje consciente de las estrategias pragmáticas-atenuantes a través del análisis de muestras de habla extraídas de un corpus discursivo oral real.

c. Entrenar al análisis del contexto comunicativo y de los interlocutores y, en función de ello, elegir las tácticas atenuantes más adecuadas.

Tras haber definido los objetivos generales y los objetivos específicos, hemos procedido al diseño de la actividad didáctica que ha sido concebida, organizada y ejecutada en 6 fases:

Fase 1: Presentación de las preguntas con necesidad de respuestas atenuadas

Fase 2: Definición de atenuación e identificación de elementos atenuantes

Fase 3: Lista de tácticas atenuantes

Fase 4: Identificación y análisis de elementos atenuantes en la trascripción de una muestra extraída de un corpus oral real

Fase 5: Revisión de las clasificaciones de los elementos atenuantes propuestas por los estudiantes

Fase 6: Clasificación y explicación de las categorías y elementos atenuantes no identificados por los estudiantes.

\section{Desarrollo de la actividad didáctica}

Se describen a continuación las 6 fases de la actividad didáctica.

Fase 1: Preguntas sobre situaciones que necesitan respuestas atenuadas.

Se ha entregado a los estudiantes seis preguntas elaboradas por el profesor, sobre diferentes situaciones a las que tenían que contestar por escrito, como si la interacción fuese oral, teniendo en cuenta tanto del contexto comunicativo concreto en el que tenía lugar la interacción como del interlocutor. Las preguntas han sido formuladas de tal manera que, se supone, los estudiantes deberían contestar de manera atenuada. 


\section{Preguntas y respuestas de los estudiantes ${ }^{2}$}

Se han marcado en azul las respuestas atenuadas y en rojo las respuestas no atenuadas.

Pregunta 1: Estás en la universidad y estás hablando con una amiga, que también es tu compañera de clases, sobre el aborto, ya que otra amiga vuestra está embarazada y no sabe si quiere o no tener un hijo. Tu y tú amiga tenéis opiniones opuesta sobre el tema. Decide si estás a favor o en contra del aborto e imagina que estáis discutiendo sobre ello. ¿Qué le dices para que cambie de opinión? ¿Qué le dices para convencerla de que tu punto de vista es el correcto?

- No lo sé pero si yo tengo dudas no estoy segura que me gustaría tener un hijo.

- ¿No te parece malo tener un hijo si no lo quieres? Eso no se hace.

- Yo creo/ Yo $\mathrm{creo}^{3}$ que hay que pensarlo bien, porque si no lo quiero en el futuro el niño va a sufrir.

- Yo estoy a favor del aborto, si me pasara lo mismo, probablemente abortaría.

- Eres egoísta, porque tú no estás pensando en la situación como si fueras tú, estás pensando en la teoría.

- Piénsalo bien ¿no crees que no tiene que ser fácil elegir? Yo creo/ Yo creo que en esa situación abortaría seguro.

- Tienes razón pero a mí no me gustaría tener un hijo que no quiero.

Pregunta 2: Estás haciendo la cola en el supermercado para pagar, estás esperando tu turno y de repente una persona mayor se mete en la cola delante de ti. ¿Qué le dices?

- Perdona pero estaba yo antes y no me ha preguntado si podía pasar.

- Señora, tengo prisa y estaba antes, por favor... / por favor... ${ }^{4}$

- Señor, hay que respetar la cola, todo estamos esperando para pagar...

- Si quiere pasar, pero yo estaba antes que Usted..

- Yo respeto los ancianos pero se debería preguntar por favor...

Pregunta 3: Tus mejores amigos te proponen ir a cenar con ellos en un restaurante pero también han invitado otro amigo que ellos consideran muy simpático pero que tú no soportas, y decides que no quieres ir. ¿Qué le dices?

- No sé si puedo ir a la cena porque ya había invitado a mis padres para cenar.

- Yo creo que no voy a ir en ese restaurante porque me han dicho que no se come muy bien.

- No sé si voy a ir, porque estoy un poco cansada, pero os lo digo más tarde.

- Me gustaría ir pero esta Carlos y no vamos muy de acuerdo ${ }^{5}$

- Yo no voy porque está Pablo y ya sabes que para mí es antipático. Otra vez voy si no está él.

Pregunta 4: Estás estudiando en la biblioteca pero a tu lado hay un chico al que le suena cada 5 minutos el móvil, es muy molesto y no consigues concentrarte. ¿Qué le dices?

- Perdona, estamos en la biblioteca ¿puedes quitar el sonido al teléfono?

${ }^{2}$ Por cuestiones de espacio se incluyen en el mismo documento tanto las preguntas que han sido entregadas a los estudiantes como una muestra representativa de sus respuestas.

${ }^{3}$ Hemos marcado las respuestas que incluyen el "yo creo" tanto con función atenuantes como función intensificadora ya que, de acuerdo con Soler, (2016), el valor de los verbos doxásticos de opinión pueden alternar entre la atenuación y la intensificación en función de la naturaleza del predicado que dicho verbo introduzca.

${ }^{4}$ Puesto que la respuesta del estudiante es escrita y no sabemos con qué entonación lo diría, y que tampoco es deducible por lo que dice anteriormente, hemos marcado el "por favor..." tanto con función atenuante como sin esta.

${ }^{5}$ Calco del francés. En español equivaldría a No me llevo muy bien con él. 
- Por favor, estoy estudiando ¿puedes apagar el móvil?

-¿Por favor, puedes ir fuera con el móvil?

- Estamos estudiando y aquí no se puede usar el teléfono con el sonido.

Pregunta 5: Estás en el gimnasio, el entrenador ha abierto la puerta y todas las ventanas y tú tienes mucho frío. ¿Qué le dices?

- Tengo un poco de frío ¿se pueden cerrar las ventanas?

- ¿Podemos cerrar las ventanas por favor?

- ¿Vamos a dejar las ventanas abiertas todo el curso o se pueden cerrar?

- ¿Puedes cerrar las ventanas o la puerta? porque hace mucho frío, por favor

- Creo que hace un poco frío. Si todos tienen frío ¿podemos cerrar las ventanas? Por favor.

Pregunta 6: Tu profesor se ha equivocado en el cálculo de tu nota de examen, estás seguro de que hay un error y quieres que ella lo corrija. ¿Qué le dices?

- Profesor, creo que hay un error en mi nota ¿podemos ver mi examen?

- Profesor, perdone pero [la estudiante pregunta cómo decir désolé mais en español, que se traduce con perdone pero) creo que la nota no está bien, esta respuesta creo que es correcta.

- Profesor, creo que hay un error in mi nota ¿tiene un momento para mirar mi examen?

- Profesor, me parece que hay un error en mi nota, el total es diferente.

Fase 2: Definición de atenuación e identificación de elementos atenuantes.

Se ha entregado a los estudiantes una definición de atenuación y algunos de sus valores.

La atenuación es una estrategia pragmática (comunicativa) originada por necesidades de imagen y dirigida a mitigar y minimizar la intensidad de lo que se expresa reduciendo la fuerza ilocutiva del acto de habla, y en ocasiones, a través de mecanismos de lenguaje vago por los que se difumina o minimiza el contenido proposicional. La atenuación es, al mismo tiempo, una actividad argumentativa que permite a los hablantes formular un menor compromiso hacia lo dicho y así lograr más eficazmente las metas conversacionales de los participantes en el discurso.

Albelda (2016: 30)

Algunos de los valores de la atenuación pueden ser suavizar el mensaje, quitar relieve, mitigar, reparar, esconder la verdadera intención.

Los estudiantes han tenido que analizar si en sus respuestas había elementos atenuantes. $\mathrm{Si}$, según ellos, los había, tenían que formular una hipótesis de su función.

Fase 3: Tácticas atenuantes.

Se ha entregado a los estudiantes una lista de táctica atenuantes ${ }^{6}$ (extraídas de Albelda et alii 2014), y algunos ejemplos de sus funciones, que posteriormente han usado para analizar sus respuestas e identificar (si los había) a qué categoría pertenecían los elementos atenuantes que habían empleado en sus respuestas.

Lista de tácticas atenuantes:

Táctica 1. Modificadores morfológicos internos: sufijos, diminutivos

\footnotetext{
${ }^{6}$ Para la consulta de la lista completa que incluye los 22 factores de análisis de la atenuación se remite a Albelda et alii (2014): "Ficha metodológica para el análisis pragmático de la atenuación en corpus discursivos del español", en Oralia 17, pp. 7-62.
} 
Táctica 2. Modificadores externos: cuantificadores minimizadores, aproximativos o difusores significativos proposicionales o extraproposicionales

Táctica 3. Empleo de palabras en otra lengua

Táctica 4. Usos modalizadores ("dislocados") de los tiempos verbales

Táctica 5. Verbos, construcciones verbales y partículas discursivas modales que expresan opinión en forma de duda o de probabilidad

Táctica 6. Verbos, construcciones verbales y partículas discursivas que expresan fingimiento de incertidumbre, de incompetencia o de ignorancia

Táctica 7. Construcciones acotadoras de la opinión a la propia persona o a un determinado ámbito

Táctica 8. Peticiones, preguntas, mandatos u órdenes expresadas de forma indirecta

Táctica 9. Expresiones de disculpa

Táctica 10. Construcciones justificadoras o de excusa

Táctica 11. Impersonalizaciones

Táctica 12. Impersonalizaciones II a través del recurso al estilo directo

Táctica 13. Movimiento de reformulación

Táctica 14. Concesividad

Táctica 15. Partículas discursivas modales de objetivación

Táctica 16. Partículas discursivas y expresiones de control de contacto con el interlocutor

Táctica 17. Elementos prosódicos, paralingüísticos o gestuales: risas; alargamiento vocálico; subido o bajada de tono.

\section{Resultados de las respuestas de los estudiantes}

Tácticas de atenuación empleada por los estudiantes en sus respuestas

Táctica 2. Modificadores externos: cuantificadores minimizadores, aproximativos o difusores significativos proposicionales o extraproposicionales (un poco, algo, algo así, como, o algo, o eso, solo, y eso, no mucho, simplemente, prácticamente, en plan, más o menos, aproximadamente, de alguna manera, por así decirlo, en principio, digamos, etc.).

- No sé si voy a ir, porque estoy un poco cansada, pero os lo digo más tarde.

Táctica 5. Verbos, construcciones verbales y partículas discursivas modales que expresan opinión en forma de duda o de probabilidad (creer, parecer, ser posible, poder, imaginar, a lo mejor, quizás, tal vez, probablemente, posiblemente). Se incluye en esta variable también la atenuación que se encuentra en el eje de la modalidad deóntica (ser conveniente, ser necesario).

- Profesora, creo que hay un error en mi nota ¿podemos ver mi examen?

- Profesora, perdone pero creo que la nota no está bien, esta respuesta creo que es correcta. 
- Profesora, creo que hay un error in mi nota ¿tiene un momento para mirar mi examen?

Táctica 6. Verbos, construcciones verbales y partículas discursivas que expresan fingimiento de incertidumbre, de incompetencia o de ignorancia sobre lo que alguien sabe o es capaz (no saber, no estar seguro, seguramente, etc.).

- No sé si voy a ir, porque estoy un poco cansada, pero os lo digo más tarde.

- No sé si puedo ir a la cena porque ya había invitado a mis padres.

- Creo que hace un poco frío. Si todos tienen frío ¿podemos cerrar las ventanas? Por favor.

- No lo sé pero si yo tengo dudas no estoy segura que me gustaría tener un hijo.

Táctica 7. Construcciones acotadoras de la opinión a la propia persona (en mi opinión, a mi parecer, a mi modo de ver, que yo sepa, para mí, creo yo, digo yo, yo, etc.), o a un determinado ámbito o espacio personal (por lo menos en mi pueblo).

- Piénsalo bien ¿no crees que no tiene que ser fácil elegir? Yo creo que en esa situación abortaría seguro.

- Profesor, me parece que hay un error en mi nota, el total es diferente.

Táctica 8. Peticiones, preguntas, mandatos u órdenes expresadas de forma indirecta (o con cierto grado de indirección), y con fórmulas ritualizadas como por favor.

- Por favor, estoy estudiando ¿puedes apagar el móvil?

Táctica 10. Construcciones justificadoras o de excusa es que, porque, como, que [causal], lo que pasa es que, etc. Se incluyen las llamadas "causales de la enunciación" y expresiones justificadoras del decir como por así decirlo, por decirlo de alguna manera, es un decir.

- ¿Puedes cerrar las ventanas o la puerta? porque hace mucho frío, por favor

Táctica 11. Impersonalizaciones

1. Apelar al juicio de la mayoría o a un interlocutor general mediante pronombres (se, uno, tú, nosotros inclusivo)

2. Apelar al juicio de la mayoría mediante formas verbales impersonales, formas de despersonalización del origen deíctico del enunciado (por lo que dicen, según cuentan, por lo visto, al parecer, según parece).

- Yo creo que no voy a ir en ese restaurante porque me han dicho que no se come muy bien.

- Yo respecto los ancianos pero se debería preguntar por favor...

Táctica 14. Concesividad

1. Movimientos concesivos-opositivos que minimizan la disconformidad: sí, ... pero; no, pero; no, pero sí; no es que... pero; no, tienes razón; bueno... pero; vale... pero.

-Tienes razón pero a mí no me gustaría tener un hijo que no quiero.

Táctica 16. Partículas discursivas y expresiones de control que buscan y solicitan el consentimiento del interlocutor (¿no?, ¿vale?, ¿eh?, ¿qué te parece?, ¿sabes?), que le dan opciones (¿o qué?), que buscan su alianza o que minimizan desacuerdos o disconformidades.

-¿No te parece malo tener un hijo si no lo quieres? Eso no se hace. 
Táctica17. Elementos prosódicos, paralingüísticos o gestuales: risas; alargamiento vocálico; subido o bajada de tono risas; movimientos de los dedos entrecomillando una expresión, muecas, etc. También se incluyen ciertos usos onomatopéyicos-atenuantes.

- Señora, tengo prisa y estaba antes, por favor...

Fase 4: Identificación y análisis de elementos atenuantes en la trascripción de una muestra extraída de un corpus oral real.

Se ha entregado a los estudiantes un fragmento de la transcripción de la grabación de una conversación argumentativa informal sobre el aborto (anexo 1), extraído de un corpus oral real (inédito), en la que tenían que identificar y subrayar los elementos que según ellos eran atenuados y los que no lo eran pero que deberían haberlo sido $^{7}$, justificando, para ambos casos, el por qué de su elección.

Fase 5: Revisión de las clasificaciones de los elementos atenuantes propuestas por los estudiantes.

Tras una puesta en común con los estudiantes, el profesor ha revisado la exactitud de los análisis hechos por los estudiantes (marcados en el anexo 1), y, en los casos necesarios, ha procedido con la corrección y explicación de los errores.

Fase 6: Clasificación y explicación de las categorías y elementos atenuantes no identificados.

El profesor ha guiado los estudiantes en la identificación y clasificación de los mecanismos atenuantes que no han sido capaces de identificar y/o clasificar en la transcripción (anexo 1), explicándoles también la finalidad con la que se emplean.

\section{Resultados generales y discusión}

Resultados del análisis de la transcripción de la conversación argumentativa informal

Elementos atenuantes identificados por parte de los estudiantes:

- yo pienso (línea 3, 4, 19, 22)

- entiendo lo que dices peroo (línea 8)

- bueno (línea 15)

- ya pero (línea 19)

- para MÍI (línea 8)

Elementos atenuantes no identificados por parte de los estudiantes:

- no sabemos (línea 9, 10)

- a lo mejor (línea 10)

- bueno/ no- no sabemos (línea 10)

- o sea (línea 13)

- sí pero (línea 13)

\footnotetext{
${ }^{7}$ Elementos que atacan a la imagen del interlocutor.
} 
- hombre (línea 16)

- sí pue-/ hay niños que no tienen padres/ pero (22-23)

Falta de atenuación identificada por parte de los estudiantes:

- no estoy de acuerdo contigo (línea 8)

- yo pienso que es una bue- muy mala idea (línea 17-18)

Falta de atenuación no identificada por parte de los estudiantes:

- tú no puedes dejar a un niño puees/ morir (línea 7)

-¡no! (línea 17)

Elementos atenuantes más frecuentes, identificados por parte de los estudiantes

- Concesividad: movimientos concesivos-opositivos que minimizan la disconformidad: sí... pero

- Construcciones acotadoras de la opinión a la propia persona o a un determinado ámbito o espacio personal: para mí

- Movimiento de reformulación con partícula: bueno

- Verbos, construcciones verbales y partículas discursivas modales que expresan opinión en forma de duda o de probabilidad: yo pienso

Interpretación de los datos en cuanto a la identificación y al uso de la atenuación:

Los estudiantes saben reconocer y utilizar algunas de las tácticas de atenuación para la salvaguarda del yo-tu (imagen y protección de la imagen), prevención/reducción de conflictos, de desacuerdo o de rechazo. La función del atenuante más frecuente en sus respuestas ha sido la prevención. El análisis del juicio pragmático sobre el uso del atenuante nos indica que los informantes saben emplear y reconocer algunos elementos y mecanismos atenuantes, pero sus habilidades pragmáticas son limitadas.

Los resultados a los que llegamos, tras la observación de las tareas realizadas por los aprendices en las diferentes fases de la propuesta didáctica, nos ha permitido averiguar las capacidades atenuantes de los estudiantes.

\section{Conclusiones}

Esta actividad didáctica ha tenido varios objetivos: por un lado, comprobar los conocimientos y las habilidades atenuantes del grupo de discentes analizados, por otro lado, ampliar y fortalecer sus conocimientos pragmáticos-atenuantes a través de un análisis de una muestra real de habla. Los resultados obtenidos muestran la necesidad de emplear corpus discursivos reales como instrumento de aprendizaje de la atenuación. Gracias a ello, y la propuesta didáctica diseñada a partir de corpus, se pretende que los estudiantes de E/LE adquieran de manera consciente los mecanismos pragmáticosatenuantes para que puedan emplearlos adaptándolos a los diferentes contextos comunicativos en los que interactúan y a en función de sus interlocutores. 


\section{Bibliografía}

Albelda MARCO, Marta (2016). «Sobre la incidencia de la imagen en la atenuación pragmática». En Mihatsch, W. y M. Albelda (eds.); Revista Internacional de Lingüística (RILI) 27, 19-32.

Albelda MArco, Marta y Fernández Colomer, Ma José (2006). La enseñanza de los registros lingüisticos en E/LE. Una aplicación a la conversación coloquial. Revista de Didáctica. Recuperado el 20/02/18, de: www.marcoele.com/num/3/0218f5989b0f06708 /coloquial.pdf

AlBelda MARCO, Marta et alii (2014). «Ficha metodológica para el análisis pragmático de la atenuación en corpus discursivos del español. (ES.POR.ATENUACIÓN)». Oralia, $17,7-62$.

BAllestero Martín, Francisco José (2002). «Mecanismos de atenuación en español e inglés: implicaciones pragmáticas en la cortesía». Círculo de lingüística aplicada a la comunicación, 11. Recuperado el 03/03/2018, de http://www.ucm.es/info/circulo/no11 /ballesteros.htm

BRIZ GómEZ, Antonio (2003). «La estrategia atenuadora en la conversación cotidiana española». En D. Bravo (ed.); Actas del Primer Coloquio Edice: La perspectiva no etnocentrista de la cortesía: identidad sociocultural de las comunidades hispanohablantes (pp. 17- 46). Estocolmo: Universidad de Estocolmo.

BRIZ GómeZ, Antonio y Marta AlbeldA MARCO (2013). «Una propuesta teórica y metodológica para el análisis de la atenuación lingüística en español y portugués: La base de un proyecto en común (ES.POR.ATENUACIÓN)». Onomázein: Revista de lingüística, filología y traducción de la Pontificia Universidad Católica de Chile, 28, 288-319.

BRIZ GómEZ, Antonio y GRUPO VAL.Es.Co. (2002). Corpus de conversaciones coloquiales. Anejos de la revista Oralia.

CANALE, Michael (1983). «From communicative competence to communicative language pedagogy». Language and communication, 1(1) (pp. 1-47).[Trad. esp.] (1995). «De la competencia comunicativa a la pedagogía comunicativa del lenguaje». En M. Llobera (coord.); Competencia comunicativa: documentos básicos en la enseñanza de lenguas extranjeras (pp. 63-82). Madrid: Edelsa.

CONSEJO DE EUROPA (2001). Marco Común Europeo de Referencia para el aprendizaje, la enseñanza y la evaluación de lenguas. Instituto Cervantes-Ministerio de Educación, Cultura y Deporte. Madrid: Anaya.

CONTRERAS FERNÁNDEZ, Josefa (2012). «¿Hay diferencia en las estrategias de atenuación en los correos electrónicos españoles y alemanes?». En Miradas multidisciplinares a los fenómenos de cortesía y descortesía en el mundo hispánico. Barranquilla: Universidad del Atlántico-Programa EDICE, 451-471.

CRESTI, Emanuela y Massimo MONEGLIA (Eds.) (2005). C-ORAL-ROM: integrated reference corpora for spoken romance languages (Vol. 15). Amsterdam: JohnBenjamins Publishing.

FÉLIX-BRASDEFER, J. César (2008). «Teaching pragmatics in the classroom: Instruction of mitigation in Spanish as a Foreign Language». Hispania, 89, 479-494. 
FÉLIX-BRASDEFER, J. César (2004). «La mitigación en el discurso oral de mexicanos y aprendices de español como lengua extranjera». En D. Bravo, y A. Briz (eds.); Pragmática sociocultural: estudios sobre el discurso de cortesía en español (pp. 285302). Madrid: Ariel.

SOLER BonAfont, M. Amparo. (2016). «La función atenuante en los verbos doxásticos del español». Revista Internacional de Lingüistica Iberoamericana (RILI), XIV (27), 75-90.

\section{Anexos}

Anexo 1: Fragmento de la transcripción de la conversación argumentativa informal

Tema: el aborto; registro: informal; participantes: A, mujer $<25$ (a favor de aborto); B, mujer $<25$ (en contra del aborto).

Por cuestiones de espacio, aquí presentamos en el mismo documento (Imagen 1) tanto el fragmento de la transcripción que ha sido entregado a los estudiantes, como la totalidad de los elementos atenuados (señalados en azul), y de los no atenuados -que deberían haber sido atenuados- (señalados en rojo), marcados por el profesor y los marcados por los estudiantes. Posteriormente, en el apartado de los resultados, indicaremos los elementos atenuantes y los elementos con falta de atenuación que los estudiantes han sido capaces de identificar y los que no, con la referencia al número de línea de la transcripción.

A: bueno emm/ sobre el abortoo/ ¿tú qué opinaas?/ ¿tú piensas que es una buena idea autorizar el aborto en Francia//? por ejemplo

B: bueno/ yo pienso que puede ser una buena idea// pero depende las condiciones// emm por ejemplo yo pienso que podríaa ser legalizados peroo/ en caso en quee $\rightarrow$ la madre/// bueno huígan de la madre/ estaría en peligro peroo $\rightarrow$ del resto yo pienso que hay que darle la vida al niño// que pase lo que pase porque es algoo $\rightarrow$ es que- buenoo es algo $\rightarrow$ natural / y es algo que paso/ y eso no sée $\rightarrow / /$ o sea por ley y por/ emmpor- por amor/ tú no puedes dejar a un niño puees/ morir

A: entiendo lo que dices ${ }^{3}$ peroo no estoy de acuerdo contigo// para Mí/ la mujer tiene que tener/ tiene quee poder elegiir/ entre las dos opciones/ no sabemos lo que ha pasado antes/ no sabemos si el- bueno el padre/ está presente o no está presente/ a lo mejor /// emm bueno/ no- no sabemos el pasado de la mujer/ no podemos decir para ella que guarde el bebé sin- sin saber lo que pasó $\S$

B:

$\S$ ya peroo el niño no vaa- no tiene que pagar por los errores sus papas/ o sea/ no es porque tus papas hicieron un error/ que tú tienes que pagar tu vida por eso $\S$

A

$\S$ buenoo/ ¿y sii- y si el niño nace/ yy- y qué pasa? y no tiene familia/ noo- su familia no está- no noo/ hombre/ o no está feliz/ no- no tiene padres/ su madre no puede educarlo/ no puedee/ a lo mejor es- bueno a lo mejor sería/// una buena ideaa quee $\rightarrow$ / ¡no! yo no ${ }^{4}$ - yo pienso que es una bue- muy mala idea/ [y]

B:

[ya] peroo yo pienso que siempre se encuentran soluciones/ aunquee hay muchos niños que nacen sin- sin padres siempre/ hay familias que puede ayudar/ siempree hayy establecimientos que pueden también ayudaar/ la religión católica que puede también ayudar muchíisimo/ emm yo pienso que un niño/ cuando nace nunca está solo yy $\rightarrow$ sí pue-/ hay niños que no tenen padres/ pero eso no quiere decir que más tarde su vidaa $\rightarrow$ estaráa/ [o seaa $\rightarrow$ ]

Imagen 1. Fragmento de la transcripción de una grabación de una conversación argumentativa informal 